Document downloaded from:

http://hdl.handle.net/10251/49342

This paper must be cited as:

Jozsa, CM.; Domene Oltra, F.; Vidal Maciá, AM.; Piñero Sipán, MG.; González Salvador, A. (2014). High performance lattice reduction on heterogeneous computing platform. Journal of Supercomputing. 70(2):772-785. doi:10.1007/s11227-014-1201-2.

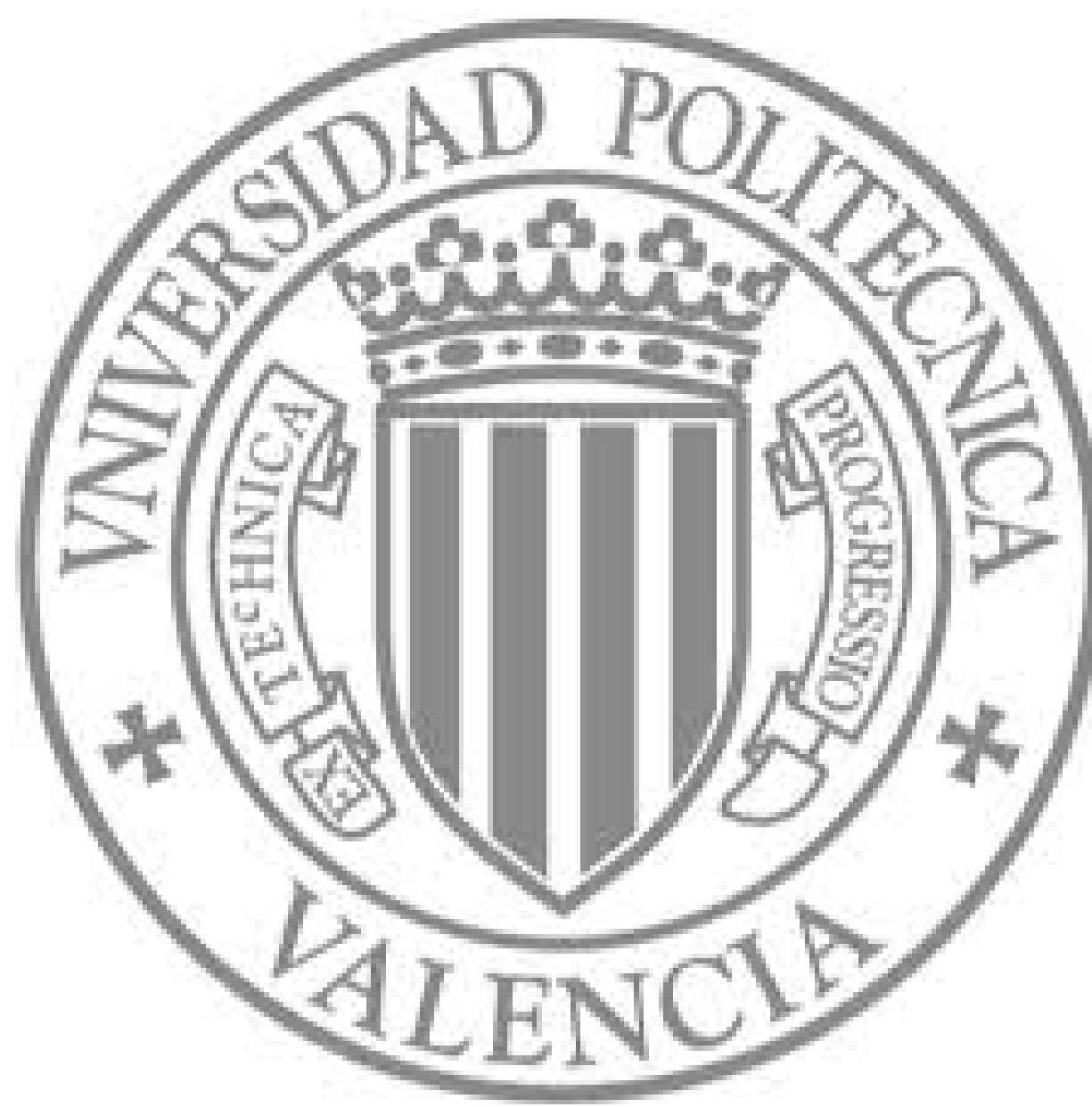

The final publication is available at

http://dx.doi.org/10.1007/s11227-014-1201-2

Copyright Springer Verlag (Germany) 


\title{
High Performance Lattice Reduction on Heterogeneous Computing Platform
}

\author{
Csaba M. Józsa • Fernando Domene • \\ Antonio M. Vidal • Gema Piñero . \\ Alberto González
}

Received: date / Accepted: date

\begin{abstract}
The lattice reduction (LR) technique has become very important in many engineering fields. However, its high complexity makes difficult its use in real-time applications, especially in applications that deal with large matrices. As a solution, the Modified Block LLL (MB-LLL) algorithm was introduced in [10], where several levels of parallelism were exploited: (i.) coarse-grained parallelism was achieved by applying the block-reduction concept presented in [15] and (ii.) fine-grained parallelism was achieved through the Cost Reduced All-Swap LLL (CR-AS-LLL) algorithm introduced in [10].

In this paper, we present the Cost Reduced MB-LLL (CR-MB-LLL) algorithm, which allows to significantly reduce the computational complexity of the MB-LLL by allowing the relaxation of the first LLL condition while executing the LR of submatrices, resulting in the delay of the GS coefficients update and by using less costly procedures during the boundary checks. The effects of complexity reduction and implementation details are analyzed and discussed for several architectures. A mapping of the CR-MB-LLL on a heterogenenous platform is proposed and it is compared with implementations running on a dynamic parallelism enabled GPU and a multi-core CPU. The mapping on the architecture proposed allows a dynamic scheduling of kernels where the overhead introduced is hidden by the use of several CUDA streams. Results show that the execution time of the CR-MBLLL algorithm on the heterogeneous platform outperforms the multi-core CPU and it is more efficient than the CR-AS-LLL algorithm in case of large matrices.
\end{abstract}

Keywords Lattice Reduction · LLL · GPU · CUDA · OpenMP

Csaba M. Józsa

Faculty of Information Technology, Pázmány Péter Catholic University, Hungary

E-mail: jozsa.csaba@itk.ppke.hu

Fernando Domene, Gema Piñero, Alberto González

Inst. Telecommunications and Multimedia Applications (iTEAM), Universitat Politècnica de València, Spain

E-mail: \{ferdool,gpinyero,agonzal\}@iteam.upv.es

Antonio M. Vidal

Dept. of Information Systems and Computation, Universitat Politècnica de València, Spain

E-mail: avidal@dsic.upv.es 


\section{Introduction}

The application of lattice reduction (LR) as a preconditioner of various signal processing algorithms plays a key role in several fields: communications, cryptography, image processing, etc. Given a basis, LR consists of finding another basis whose vectors are more orthogonal and shorter, in the sense of Euclidean norm, than the original ones. The Minkowski or Hermite-Korkine-Zolotareff reductions are the techniques that obtain the best performance in terms of reduction, but also the ones with a higher computational cost. Both techniques require the calculation of the shortest lattice vector, which has been proved to be NP-hard (see [17] and references therein).

In order to reduce the computational complexity of LR techniques, a polynomial time algorithm was proposed by Lenstra, Lenstra and Lovász, known as the LLL algorithm [11]. This algorithm can be seen as a relaxation of HermiteKorkine-Zolotareff conditions [4] or an extension of Gauss reduction [17] and obtains the reduced basis by applying two different operations over the original basis: size-reduction (linear combination between columns) and column swap. Although further reduction techniques have been proposed afterwards, the LLL algorithm is the most used due to the good trade-off between performance and computational complexity.

Regarding the hardware implementation of the LLL algorithm, several solutions can be found in the literature. Implementations that make use of LR to improve the detection performance of multiple antenna systems can be found in $[3,8,13,16]$. In [16], an LR-aided symbol detector for multiple-input multipleoutput (MIMO) and orthogonal frequency division multiple access (OFDMA) is implemented using $65 \mathrm{~nm}$ ASIC technologies. A field-programmable gate array (FPGA) implementation of a variant of the LLL algorithm, the Clarkson's algorithm, is presented in [3], whose main benefit is the computational complexity reduction without significant performance loss in MIMO detection. More recently, [8] makes use of a Xilinx XC4VLX80-12 FPGA for implementing LR-aided detectors, whereas [13] uses an efficient VLSI design based on a pipelined architecture.

In [10] low level, fine-grained parallelism was implemented by the CR-AS-LLL algorithm, where the low processing time is assured by an efficient work distribution, minimizing the idle time of the launched threads. Based on the parallel block-reduction concept presented in [15], a higher level, coarse-grained parallelism can be applied as an extra level of parallelism. The idea is to subdivide the original lattice basis matrix in several smaller submatrices and perform an independent LR on them followed by a boundary check between adjacent submatrices. The MBLLL algorithm proposed in [10] implements a parallel processing of the submatrices by using the parallel CR-AS-LLL algorithm for the LR of every submatrix.

The implementations of the previous references make use of only one architecture to calculate the LR of a basis. However, a better performance can be obtained by combining different architectures, what is known as heterogeneous computing. Among the different combinations, the use of CPU and GPU is probably the most popular since it can be found in most of the computers.

In this paper, we present the Cost Reduced MB-LLL (CR-MB-LLL) algorithm in order to further reduce the computational complexity of the MB-LLL algorithm [10]. The main idea behind the CR-MB-LLL algorithm is the relaxation of the first LLL condition while executing the LR for the submatrices, resulting 
in the delay of the GS coefficients update and by using less costly procedures when performing the boundary checks. The effects of this complexity reduction are evaluated on different architectures.

A mapping of the CR-MB-LLL algorithm on a heterogeneous platform consisting of a CPU and a GPU is proposed and it is compared with implementations running on a GPU with dynamic parallelism (DP) capability and a multi-core CPU architecture. The proposed architecture allows a dynamic scheduling of kernels where the overhead introduced by host-device communication is hidden by the use of CUDA streams. Results show that the CR-MB-LLL algorithm executed on the heterogeneous platform outperforms the DP-based GPU and multi-core implementations.

The algorithm mapping on different parallel architectures is very challenging, since the number of processing cores, latency and size of the different memory hierarchies and available cache is different. The mapping details of the CR-AS-LLL and CR-MB-LLL algorithms to different parallel architectures is also presented with a special emphasis on the work distribution among the threads and the efficient memory utilization.

The paper is organized as follows. In Section 2 a brief introduction to LR is given. The architecture dependent mapping details of the CR-AS-LLL and CRMB-LLL are presented in Section 3, followed by their performance evaluations on different platforms in Section 4. Finally, conclusions are stated in Section 5.

\section{Problem description}

A real-valued lattice $L=\left\{\sum_{i=1}^{n} x_{i} \underline{b}_{i} \mid x_{i} \in \mathbb{Z}, i=1, \cdots, n\right\}$ is a discrete additive subgroup of $\mathbb{R}^{n}$, where $\underline{b}_{1}, \underline{b}_{2}, \cdots, \underline{b}_{n} \in \mathbb{R}^{n}$ are linearly independent vectors and $\mathbb{Z}$ denotes the set of integers. Let matrix $\mathbf{B}=\left(\underline{b}_{1}, \ldots, \underline{b}_{n}\right) \in \mathbb{R}^{n \times n}$ denote the full (column) ranked basis of the lattice. Let $\mathbf{B}^{*}=\left(\underline{b}_{1}^{*}, \ldots, \underline{b}_{n}^{*}\right) \in \mathbb{R}^{n \times n}$ denote the associated orthogonal basis of $\mathbf{B}$, calculated by the Gram-Schmidt (GS) orthogonalization process as $\underline{b}_{1}^{*}=\underline{b}_{1}$ and $\underline{b}_{i}^{*}=\underline{b}_{i}-\sum_{j=1}^{i-1} \mu_{i, j} \underline{b}_{j}^{*}$ for $2 \leq i \leq n$, where $\mu_{i, j}=\left(\underline{b}_{i}, \underline{b}_{j}^{*}\right) /\left(\underline{b}_{j}^{*}, \underline{b}_{j}^{*}\right)$ for $1 \leq j<i \leq n$, also called the GS coefficients and $(\cdot, \cdot)$ denotes the ordinary dot product on $\mathbb{R}^{n}$. Thus, matrix $\mathbf{B}$ can be expressed as $\mathbf{B}=\mathbf{B}^{*} \cdot \mathbf{U}$, where matrix $\mathbf{U}$ is upper triangular with unit diagonal, and whose element $(i, j)$ above the diagonal is given by the GS coefficient $\mu_{j, i}$.

Definition 1 Given a lattice $\mathbf{L} \in \mathbb{R}^{n}$ with basis $\mathbf{B} \in \mathbb{R}^{n \times n}$, associated orthogonal basis $\mathbf{B}^{*} \in \mathbb{R}^{n \times n}$, and GS coefficients $\mu_{i, j}, \mathbf{B}$ is called $L L L$-reduced if the following conditions are satisfied:

$$
\begin{aligned}
\left|\mu_{i, j}\right| & \leq \frac{1}{2} & & \text { for } 1 \leq j<i \leq n \\
\left\|\underline{b}_{i}^{*}+\mu_{i, i-1} \underline{b}_{i-1}^{*}\right\| & \geq \delta\left\|\underline{b}_{i-1}^{*}\right\| & & \text { for } 1<i \leq n, \frac{3}{4} \leq \delta<1
\end{aligned}
$$

\section{Parallel Lattice-Reduction algorithms and their mapping to parallel architectures}

Since, the LLL algorithm shows a highly sequential behavior, multiple levels of parallelism have to be identified and exploited in order to efficiently parallelize 
this algorithm. Dividing the problem in several sub-problems that can be executed concurrently, can be regarded as one level of parallelism. In addition, if a sub-problem could benefit from a multi-threaded environment it can be regarded as a second level of parallelism. Previous parallel LR implementations, such as the ones presented in $[1,2,12]$, have focused only on multi-core architectures. The main drawback of the low number of threads offered by modern CPUs (compared to GPUs) is that low level parallelism can not be efficiently exploited. During an algorithm design, low level parallelism is usually omitted and the levels of parallelism are also restricted. In case of GPUs, the high number of CUDA cores makes possible the parallel execution of a high number of threads leading to significant performance improvements.

\subsection{Mapping details of the CR-AS-LLL algorithm}

Basically, LR consists of a succession of swaps between vectors of the basis and some operations to decrease their norms. The order in which the swaps are applied in the LLL algorithm is limiting in a parallel framework. Villard in [14] introduced the any swap reduction concept, that enables simultaneous basis swaps and served as a basis for future parallel implementations. In [12], the concept of delaying the size reductions was introduced. In the CR-AS-LLL algorithm, further computational cost is saved by rearranging and delaying the frequently used size reduction procedure.

Procedures SimpleSizeReduce, SimpleSwap and Swap are defined in order to give an accurate description of the CR-AS-LLL algorithm.

Procedure 1 (SimpleSizeReduce $\left(\mathbf{B}_{\mathbf{i}}, k, l\right)$ ) Given a lattice generator matrix $\mathbf{B}$ and the associated GS coefficients matrix $\mathbf{U}$, if the condition (1) is not satisfied, i.e. $\left|\mu_{k, l}\right|>\frac{1}{2}$, the following updates have to be applied:

- $\mu=\left\lceil\mu_{k, l}\right\rfloor, \mu_{k, l}=\mu_{k, l}-\mu, \underline{b}_{k}=\underline{b}_{k}-\mu \underline{b}_{l}$.

Procedure 2 (SimpleSwap $\left.\left(\mathbf{B}_{\mathbf{i}}, k\right)\right)$ Given a lattice generator matrix $\mathbf{B}$, the associated orthogonal basis $\mathbf{B}^{*}$ and GS coefficients matrix $\mathbf{U}$, if the condition (2) is not satisfied, or equivalently $\left\|\underline{b}_{k}^{*}\right\|^{2}<\left(\delta-\mu_{k, k-1}^{2}\right)\left\|\underline{b}_{k-1}^{*}\right\|^{2}$, the following updates have to be applied:

- $\operatorname{swap} \underline{b}_{k}$ with $\underline{b}_{k-1}$

- $\underline{b}_{k-1}^{* \text { । }}=\underline{b}_{k}^{*}+\mu_{k, k-1} \underline{b}_{k-1}^{*}, \mu_{k, k-1}^{\prime}=\left(\underline{b}_{k-1}^{*}, \underline{b}_{k-1}^{* \text { । }}\right) /\left\|\underline{b}_{k-1}^{* 1}\right\|^{2}$, $\underline{b}_{k}^{* 1}=\underline{b}_{k-1}^{*}-\mu_{k, k-1}^{\prime} \underline{b}_{k-1}^{* 1}$

- $\underline{b}_{k-1}^{*}=\underline{b}_{k-1}^{* 1}, \underline{b}_{k}^{*}=\underline{b}_{k}^{* 1}, \mu_{k, k-1}=\mu_{k, k-1}^{1}$

Procedure $3\left(\mathbf{S w a p}\left(\mathbf{B}_{\mathbf{i}}, k\right)\right)$ Given a lattice generator matrix $\mathbf{B}$, the associated orthogonal basis $\mathbf{B}^{*}$ and $G S$ coefficients matrix $\mathbf{U}$, if the condition (2) is not satisfied, or equivalently $\left\|\underline{b}_{k}^{*}\right\|^{2}<\left(\delta-\mu_{k, k-1}^{2}\right)\left\|\underline{b}_{k-1}^{*}\right\|^{2}$, the following updates have to be applied:

- perform SimpleSwap $(k)$

- $\operatorname{swap} \mu_{k, j}$ with $\mu_{k-1, j}$, for $1 \leq j<k-1$,

- $\left(\begin{array}{c}\mu_{i, k-1} \\ \mu_{i, k}\end{array}\right)=\left(\begin{array}{c}\mu_{i, k-1} \mu_{k, k-1}^{1}+\mu_{i, k}\left\|\underline{b}_{k}^{*}\right\|^{2} /\left\|\underline{b}_{k-1}^{* 1}\right\|^{2} \\ \mu_{i, k-1}-\mu_{i, k} \mu_{k, k-1}\end{array}\right)$ for $k+1 \leq i<n$. 


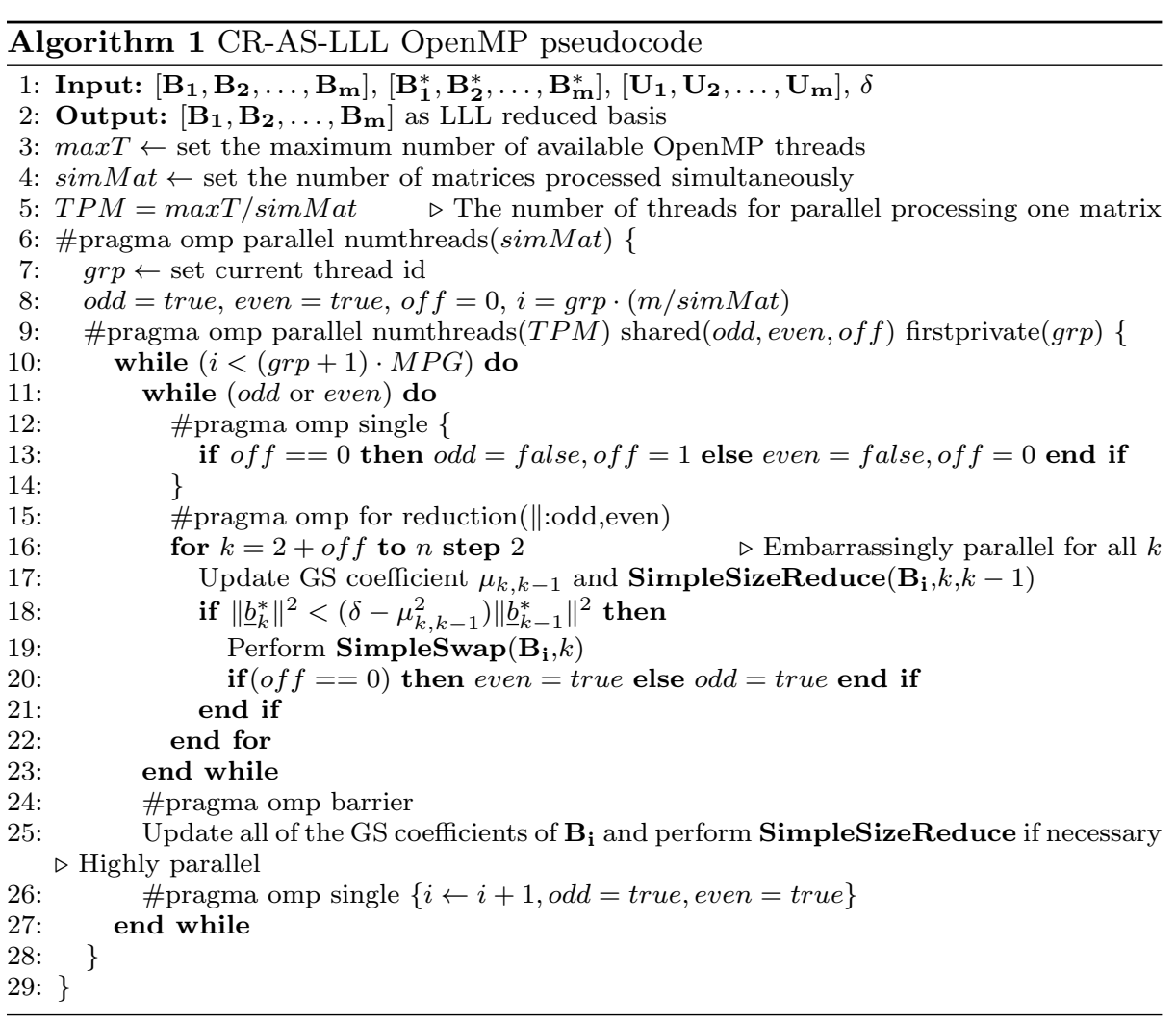

In Alg. 1, the OpenMP implementation of the CR-AS-LLL is presented. Twolevel parallelism is implemented based on a nested parallelism construct. The outer level parallelism starts the concurrent processing of simMat number of lattice basis and the inner parallel construct is responsible for the parallel LR of a basis with TPM number of threads. As the outer level parallelism is expanded, namely simMat is increased, the threads available for the parallel LR are decreased.

When mapped to the GPU, the performance of the CR-AS-LLL algorithm depends on the efficiency of the work distribution among the available GPU threads and the implementation of the most frequently used operations, such as dot products, size reductions and column swaps. In Alg. 2, the CUDA pseudo-code is presented with a two dimensional thread block $\operatorname{TB}\left(T_{x}, T_{y}\right)$ configuration, where $T_{x}$ and $T_{y}$ denotes the number of threads in the $x$ and $y$ dimension. The kernel is launched with a one dimensional grid whose size is determined by the number of basis processed simultaneously. The number of threads $T_{y}$ is defined based on the size of the original basis, i.e. $T_{y}=\max (n / 2,32)$. By enabling the usage of $T_{x}=\max (n, 32)$ threads in the $x$ dimension, the threads that belong to the same $y$ dimension will form a warp, consequently the global memory loads and stores issued by the threads of the warp will be coalesced. The $y$ dimension also defines the extent of paralellism. The iteration variable of the for loop is increased in every iteration by $T_{y} \cdot 2$. In other words, in every phase the threads with the same $i d_{y}$ have to reduce and swap at most $n /\left(T_{y} \cdot 2\right)$ vectors. 


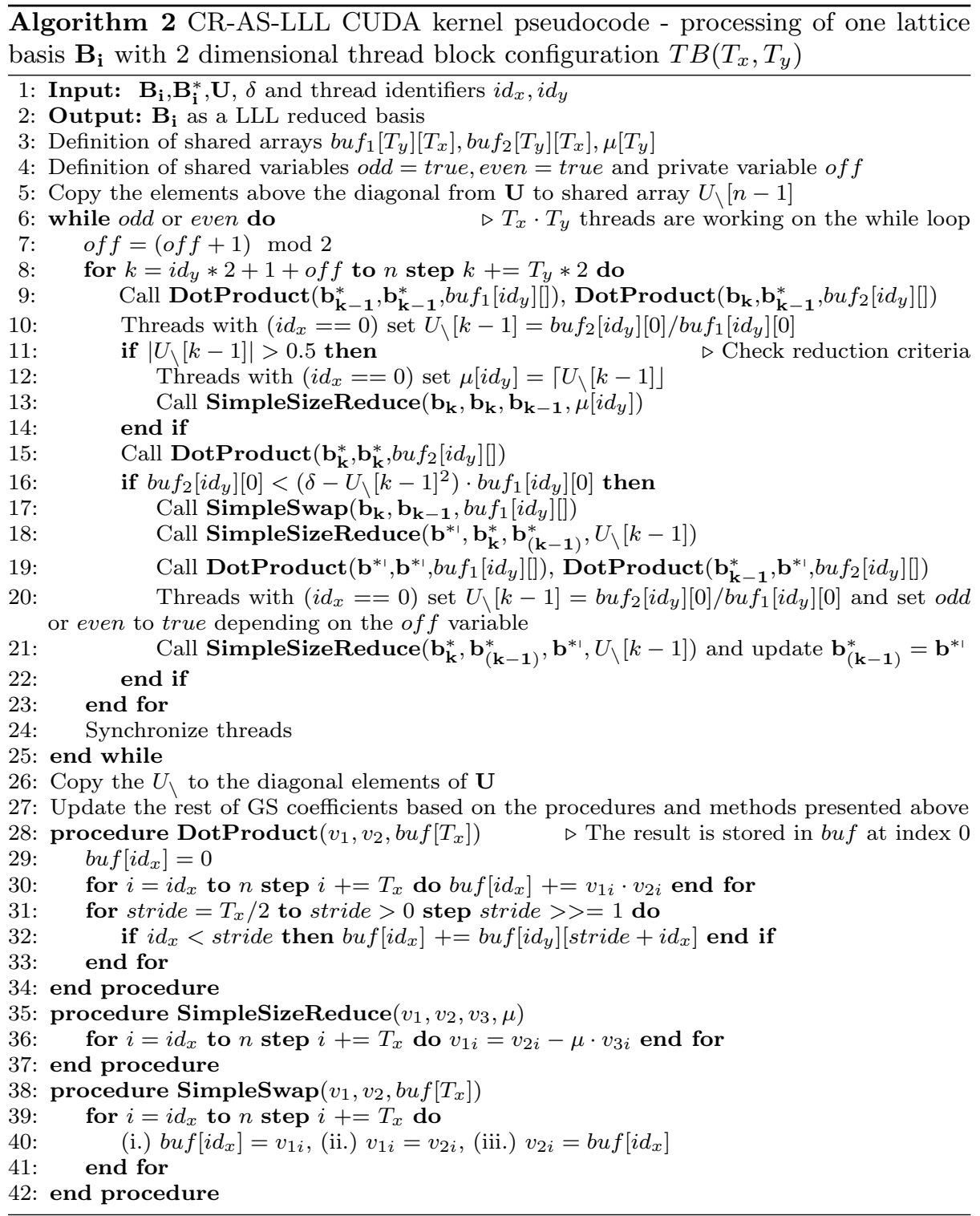

The elements of matrices $\mathbf{B}, \mathbf{B}^{*}, \mathbf{U}$ are stored in the global memory of the GPU. Since the size of shared memory is limited, it is not possible to load the entire matrices in this low latency memory. Furthermore, the excessive use of shared memory is decreasing the occupancy, resulting in performance degradation. Shared buffers bu $f_{1}\left[T_{y}\right]\left[T_{x}\right]$ and $b u f_{2}\left[T_{y}\right]\left[T_{x}\right]$ are allocated in order to efficiently compute the dot products and the vector norms. In order to avoid unnecessary access to the global memory, the GS coefficients right above the diagonal are also stored in the shared buffer $U_{\backslash}$ due to their frequent access. 
A major difference between the CUDA and OpenMP mapping lies in the implementation of the size reductions, dot products and swaps. In the CUDA implementation, because of the two dimensional TB configuration, $T_{x}$ number of threads are working in every procedure. For example, in the dot product calculation every thread has to do $n / T_{x}$ number of multiplications and the result of the multiplication is added to the shared memory buffer. When the execution of all the thread finishes, a parallel prefix sum is applied on the buffer in order to conclude the dot product computation. In case of the OpenMP implementation, only one thread is working in the computation of a dot product since the number of threads are limited.

\subsection{Mapping details of the CR-MB-LLL algorithm}

As stated in the previous sections the MB-LLL algorithm allows to split a large matrix in several smaller submatrices where parallel LR is performed in a blockwise manner with the parallel LR algorithm CR-AS-LLL. Once the LR of the submatrices is finished, the boundaries between adjacent submatrices are checked and finally the GS coefficients outside the initial groups are updated. The main condition is to keep every submatrix as an LLL-reduced matrix throughout the processing. In [10], a detailed description of the MB-LLL algorithm is shown.

The proposed CR-MB-LLL algorithm further reduces the computational complexity of the MB-LLL algorithm. In the MB-LLL algorithm, the submatrices affected by a boundary swap have to be LLL-reduced and the GS coefficients have to be updated. Moreover, in order to maintain the LLL conditions in the submatrices affected by a boundary swap, the Swap procedure has to be performed. The complexity reduction is achieved by eliminating the GS coefficients update in the submatrices after the execution of the CR-AS-LLL and the usage of the SimpleSwap procedure instead of Swap in case of a boundary swap. Since the GS coefficients are updated only when the ordering condition (2) is met for every column vector, the processing time can be considerably reduced.

In the following, algorithm mappings are proposed for a (i.) GPU, (ii.) multicore CPU architecture and (iii.) a heterogeneous system.

The GPU mapping of the CR-MB-LLL algorithm is similar to the one presented in Section 3.1, since the procedures used are performed with a two dimensional TB configuration even in the case of a boundary check. The main difference is that dynamic parallelism (DP) enables the launch of new kernels from the GPU without returning the program flow control to the CPU. DP is a feature that was introduced in CUDA 5.0 and device compute capability 3.5 is required.

The schematic of the kernels scheduling implementing the DP is shown in Fig. 1. The CPU launches the Block kernel. The size of the grid is equal to the number of matrices that are simultaneously processed and the number of threads in one $\mathrm{TB}$ is equal to the number of submatrices. In this case, every thread has to prepare the data for the corresponding submatrices and launch the $C R$ - $A S$ $L L L$ kernel. The kernel has to be relaunched if the LLL conditions were broken by a boundary swap, which can be solved by tracking state variables placed in the global memory. When all the submatrices are reduced, the Boundaries Check $(B C)$ kernel is launched. Since the operations performed in this section are dot products and column swaps, the thread configuration of the TB is the same as 


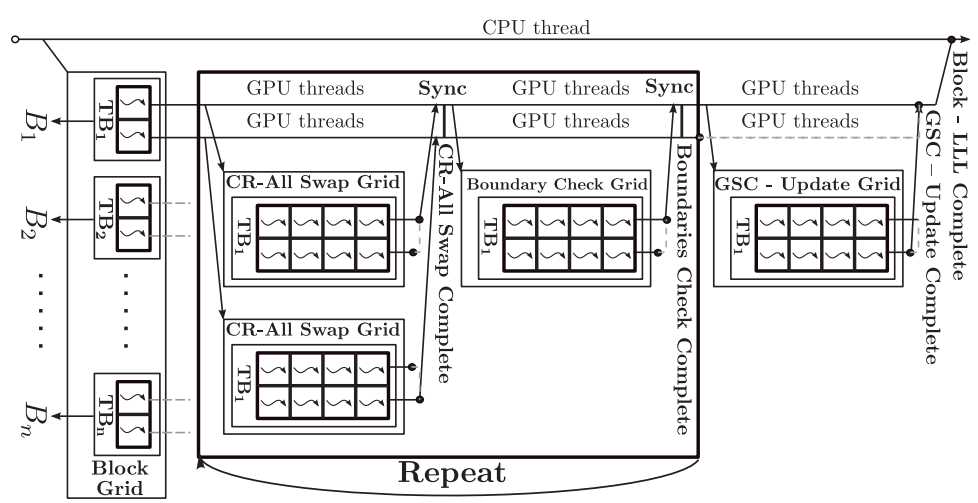

Fig. 1: Kernels scheduling on dynamic parallelism enabled GPU for the CR-MB-LLL algorithm.

in case of the $C R$ - $A S-L L L$ kernel. The $C R$ - $A S-L L L$ and $B C$ kernels are repeated until there are no swaps on the boundaries. Because one matrix is assigned to one TB in the parent Block kernel, the processing of the different matrices can be done simultaneously despite the variable number of iterations. Finally, the GS coefficients outside the blocks are updated with the GSC-Update kernel and the size-reduction is performed wherever is needed.

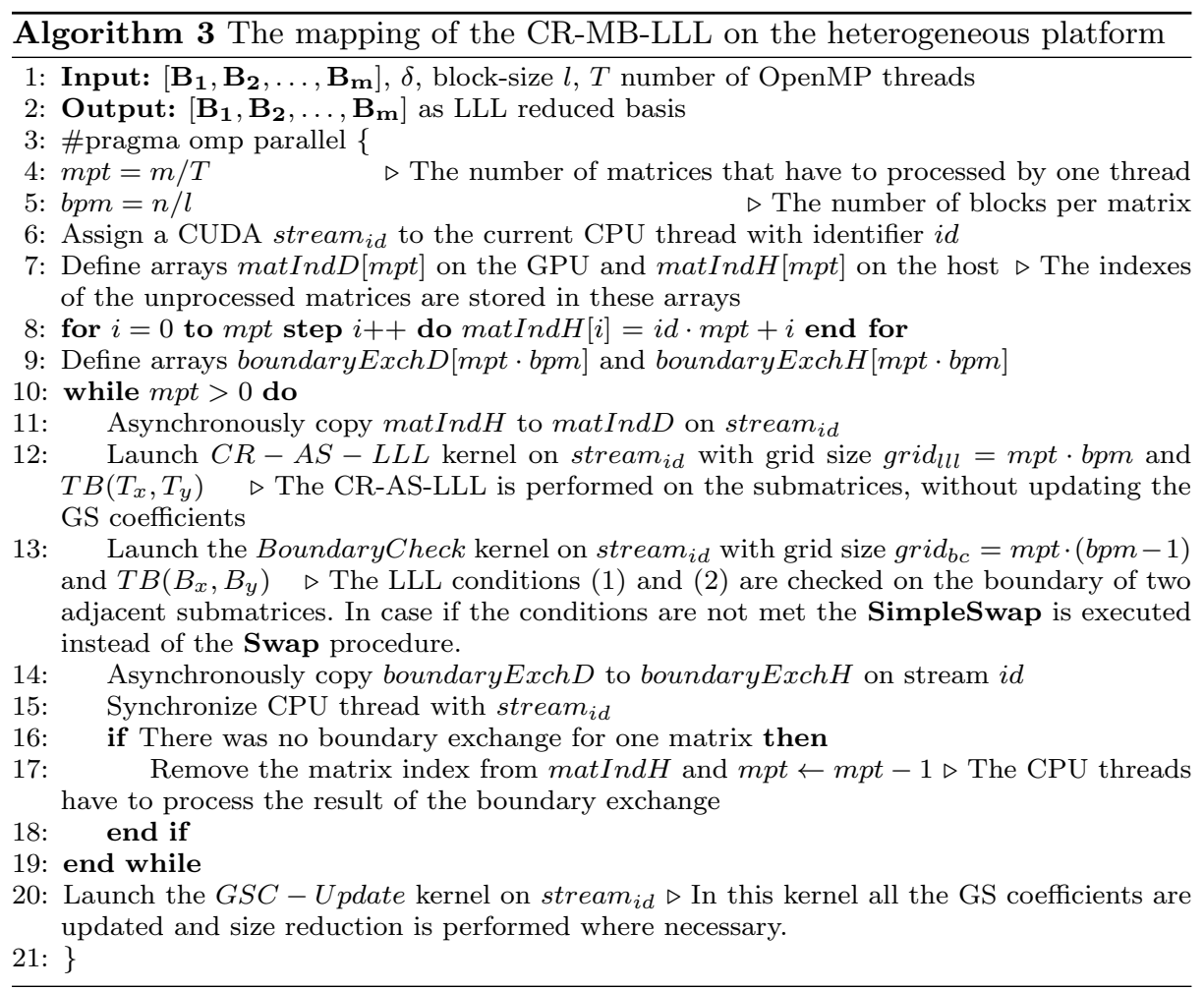




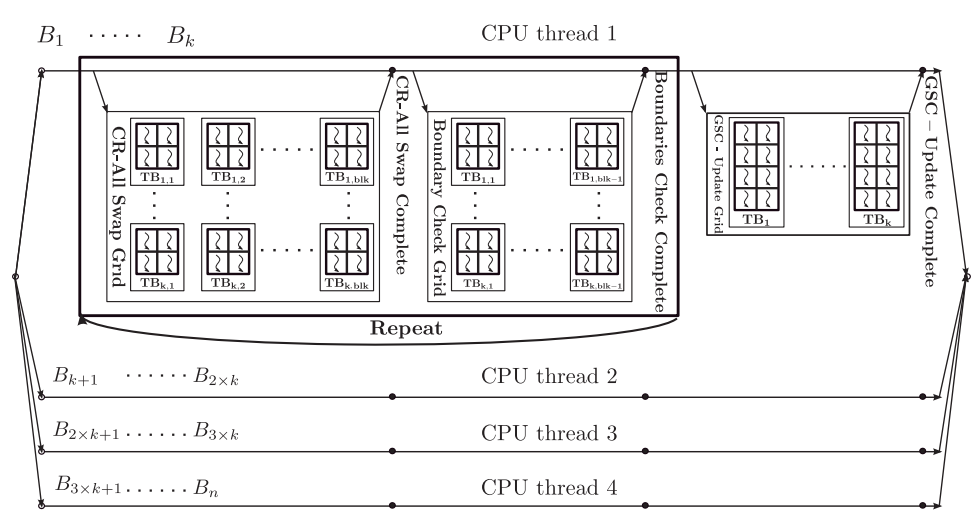

Fig. 2: Kernels scheduling on the heterogeneous platform for the CR-MB-LLL algorithm.

The schematic of the heterogeneous platform is shown in Fig. 2. The CPU threads are responsible for launching $C R$-AS-LLL, BC and GSC-Update kernels, update the state variables and implement the control logic of the dynamic scheduling. The mapping of the CR-MB-LLL algorithm on the heterogeneous platform is presented in Alg. 3.

A different CUDA stream is assigned for every CPU thread, making possible the concurrent kernel execution and reducing the idle time of the CUDA cores. Before launching the $C R$ - $A S$ - $L L L$ and $B C$ kernels, the CPU thread updates the matIndD array placed in the GPU's global memory to specify which matrices need further processing. The size of the grid is dynamically adjusted according to the number of non-processed matrices in every iteration. After the Boundary Check kernel is executed and the boundaryExchH is updated on the host, the CPU thread checks if the LR of any matrix is finished. If LLL reduced matrices are found, the matIndH is updated and consequently the size of the grids assigned to the $C R$ - $A S$ - $L L L$ and $B C$ kernels is decreased. The $G S C$-Update kernel starts after all the matrices assigned to one CPU thread have been completely processed.

The control structure required by the multi-core architecture is similar to the one presented in the heterogeneous platform. The difference is that instead of launching GPU kernels, the master threads fork a specified number of slave threads that are processing the submatrices in parallel. The parallel LR of the submatrices is performed according to $\mathrm{Alg}$. 1. In this case the very limited number of CPU threads restrict the exploitation of several levels of parallelism.

\section{Evaluation results}

In this section we present the performance results of the proposed algorithms. The computations were done in single-precision floating point arithmetic and parameter $\delta=0.75$ was used for the LLL condition (2). Block-Toeplitz matrices have been considered to evaluate the performance of the different implementations. These type of matrices are usually used in wireless communications [17].

Figure 3 shows the computational times of the MB-LLL algorithm based on the architectures discussed in Section 3.2 for different matrix dimensions, where 


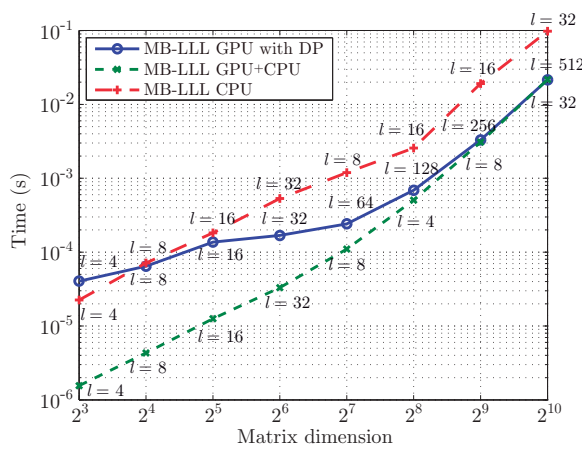

Fig. 3: Computational time of the MB-LLL algorithm on different architectures, where $l$ denotes the size of the processed blocks.

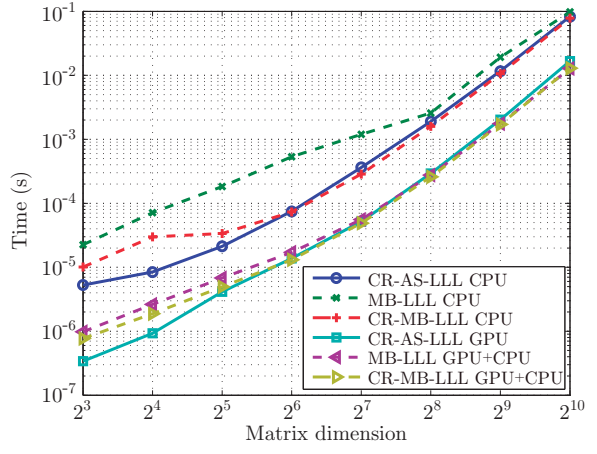

Fig. 4: Computational times of algorithms CR-AS-LLL, MB-LLL and CR-MB-LLL for matrix dimensions $2^{3}-2^{10}$

$l$ denotes the size of the processed blocks. The performance measurements were evaluated with all the possible block sizes and the best configuration is shown. The architectures used for the computational time measurements are the Nvidia Tesla K20 (DP capability) and an Intel Core i7-3820 processor. The processing times show similar performance for large matrices when the GPU is involved. However, the heterogeneous platform clearly outperforms the solution based on DP in the case of small matrices. This gap is caused by the overhead required when launching kernels from kernels with DP and the limited overlapping execution of kernels on different streams. The conclusion is that the data transfer between CPU and GPU required by the heterogeneous system is less time consuming than the overhead of the kernel launch with DP and the limitation of the concurrent execution of kernels on different streams.

Figure 4 compares the average computational time of the CR-AS-LLL, MBLLL and CR-MB-LLL algorithms for different matrix dimensions. The algorithms were evaluated on the Intel Core i7-3820 CPU, the Nvidia GeForce GTX 690 GPU and the heterogeneous system containing the previously mentioned CPU and GPU. In [7], it was shown that the GTX 690 has a better performance than the K20 when performing LR, thus the DP is not required. Regarding the GPU and comibned CPU+GPU implementations the following conclusions can be drawn: (i.) the computational time of the CR-MB-LLL is $25-40 \%$ lower in case of small and medium-sized matrices compared to the MB-LLL algorithm and the performance is similar in case of larger matrices, (ii.) the CR-AS-LLL performs better than the CR-MB-LLL in case of small matrices, however for large matrices the block concept implemented in the CR-MB-LLL achieves $30 \%$ speed-up compared to the CR-AS-LLL and (iii.) the systems using the GPU outperform the CPU for every matrix dimension with speed-ups ranging from 6 to 15 .

Regarding the CPU implementations the following conclusions can be drawn: (i.) the CR-MB-LLL always outperforms the MB-LLL algorithm with speed-ups ranging from 2 to 7 , (ii.) the CR-AS-LLL algorithm performs better than the MB-LLL and CR-MB-LLL for matrices with low dimensions $\left(2^{3}-2^{6}\right)$, (iii.) the computational time of the CR-MB-LLL is $10-20 \%$ lower in case of larger matrices compared to the CR-AS-LLL. 


\begin{tabular}{|c|c|c|c|c|c|c|}
\hline Ref & Algorithm & Architecture & $4 \times 4$ & $8 \times 8$ & $64 \times 64$ & $1024 \times 1024$ \\
\hline$[3]$ & Clarksons Algorithm & Virtex-II-Pro FPGA & $4.2 \times 10^{-6}$ & $\mathrm{x}$ & $\mathrm{x}$ & $\mathrm{x}$ \\
\hline$[9]$ & Complex LLL & Virtex-5 FPGA & $0.79 \times 10^{-6}$ & $\mathrm{x}$ & $\mathrm{x}$ & $\mathrm{x}$ \\
\hline$[6]$ & Brun's Algorithm & ASIC 250 nM & $0.07 \times 10^{-6}$ & $\mathrm{x}$ & $\mathrm{x}$ & $\mathrm{x}$ \\
\hline$[5]$ & Reverse Siegel LLL & Virtex-4 FPGA & $0.18 \times 10^{-6}$ & $\mathrm{x}$ & $\mathrm{x}$ & $\mathrm{x}$ \\
\hline$[5]$ & Reverse Siegel LLL & ASIC 130 nM & $0.04 \times 10^{-6}$ & $\mathrm{x}$ & $\mathrm{x}$ & $\mathrm{x}$ \\
\hline$[1]$ & SB-LLL & ADRES & $0.17 \times 10^{-6}$ & $\mathrm{x}$ & $\mathrm{x}$ & $\mathrm{x}$ \\
\hline This work & CR-AS-LLL & GTX690 GPU & $\mathrm{x}$ & $0.33 \times 10^{-6}$ & $1.37 \times 10^{-5}$ & $1.67 \times 10^{-2}$ \\
\hline This work & CR-MB-LLL & $\begin{array}{c}\text { GTX690 GPU + } \\
\text { Intel i7-3820 CPU }\end{array}$ & $\mathrm{x}$ & $0.77 \times 10^{-6}$ & $1.30 \times 10^{-5}$ & $1.28 \times 10^{-2}$ \\
\hline
\end{tabular}

Table 1: Performance comparison of different lattice reduction implementations.

A surprising result is that, while the CR-MB-LLL achieves a significant speedup compared to the MB-LLL for the CPU architecture, the same is not true for GPU architecture. This fact is due to several reasons. The computational complexity reductions for the CR-MB-LLL affect only the $C R-A S-L L L$ and $B C$ kernels. However, in case of large matrices, the GSC-Update kernel is taking the major part of the processing time. This kernel has to access the global memory frequently and these accesses have a high latency. In case of the CPU, this problem is alleviated by the high speed memory access and the large amount of available cache for CPU.

The performance of LR mostly depends on the precision of the computation, the size and type of the basis matrix and the architecture used. In Table 1 performance of existing implementations are presented. Previous research mostly focused on small matrices. In [2] performance measures for higher dimension matrices are presented as well, however the total runtime of the algorithm is not specified.

\section{Conclusions}

In this paper, we proposed the CR-MB-LLL algorithm and we have compared it with the CR-AS-LLL and MB-LLL algorithms presented in [10]. The idea behind the CR-MB-LLL algorithm is the relaxation of LLL condition (1) for the submatrices, resulting in the delay of the GS coefficients update when executing the LR and the replacement of the Swap procedure by the less costly SimpleSwap procedure when performing the boundary checks.

The CR-MB-LLL algorithm has been evaluated on several architectures: a multi-core architecture, a GPU with DP capability and a heterogeneous platform based on a CPU and GPU. Results show that mapping the CR-MB-LLL algorithm on the heterogeneous architecture reduces the computational time by $30 \%$ compared to the CR-AS-LLL in case of large matrices, whereas implementations involving GPUs achieve speed-up factors from 6-15 compared to the multi-core CPU architecture. The MB-LLL algorithm achieves speed-up factors ranging from $5-25$ when launched on the proposed heterogeneous platform compared to the DP-based GPU implementation for matrix dimensions ranging $2^{3}-2^{6}$.

It was shown that the efficiency of the CR-MB-LLL is significantly affected by the architectures used. The CR-MB-LLL is $1.5-7$ times faster compared to the MB-LLL algorithm when launched on multi-core CPU architecture, however the CR-MB-LLL is only at most 1.4 times faster compared to the MB-LLL when launched on the GPU architecture. This is mainly because the computational complexity reductions introduced in the CR-MB-LLL algorithm affect the $C R$ - $A S$ $L L L$ and $B C$ kernels. However, in case of large matrices the GSC-Update kernel is 
taking the major part of the processing time with frequent accesses to the global memory of the GPU. In case of the CPU, the memory access has a lower latency and the available cache for CPU is significantly bigger, causing different speed-ups of the same algorithm on the different architectures.

Acknowledgements Financial support for this study was provided by grants TÁMOP4.2.1./B-11/2/KMR-2011-0002, TÁMOP-4.2.2/B-10/1-2010-0014 from the Pázmány Péter Catholic University, European Union ERDF, Spanish Government through TEC2012-38142C04-01 project and Generalitat Valenciana through PROMETEO/2009/013 project.

\section{References}

1. Ahmad, U., Amin, A., Li, M., Pollin, S., Van der Perre, L., Catthoor, F.: Scalable blockbased parallel lattice reduction algorithm for an SDR baseband processor. In: Communications (ICC), 2011 IEEE International Conference on (2011)

2. Backes, W., Wetzel, S.: Parallel lattice basis reduction - the road to many-core. In: High Performance Computing and Communications (HPCC), 2011 IEEE 13th International Conference on (2011)

3. Barbero, L.G., Milliner, D.L., Ratnarajah, T., Barry, J.R., Cowan, C.: Rapid prototyping of Clarkson's lattice reduction for MIMO detection. In: Communications, 2009. ICC'09. IEEE International Conference on, pp. 1-5 (2009)

4. Bremner, M.R.: Lattice basis reduction: An introduction to the LLL algorithm and its applications. CRC Press (2012)

5. Bruderer, L., Studer, C., Wenk, M., Seethaler, D., Burg, A.: VLSI implementation of a low-complexity LLL lattice reduction algorithm for MIMO detection. In: Circuits and Systems (ISCAS), Proceedings of 2010 IEEE International Symposium on (2010)

6. Burg, A., Seethaler, D., Matz, G.: VLSI implementation of a lattice-reduction algorithm for multi-antenna broadcast precoding. In: Circuits and Systems, 2007. ISCAS 2007. IEEE International Symposium on, pp. 673-676 (2007)

7. Domene, F., Józsa, C.M., Vidal, A.M., Piñero, G., Gonzalez, A.: Performance analysis of a parallel lattice reduction algorithm on many-core architectures. In: Proceedings of the 13th International Conference on Computational and Mathematical Methods in Science and Engineering (2013)

8. Gestner, B., Zhang, W., Ma, X., Anderson, D.: Lattice reduction for MIMO detection: From theoretical analysis to hardware realization 58(4), 813-826 (2011)

9. Gestner, B., Zhang, W., Ma, X., Anderson, D.V.: VLSI implementation of a lattice reduction algorithm for low-complexity equalization. In: Circuits and Systems for Communications, 2008. ICCSC 2008. 4th IEEE International Conference on, pp. 643-647 (2008)

10. Józsa, C.M., Domene, F., Piñero, G., González, A., Vidal, A.M.: Efficient GPU implementation of lattice-reduction-aided multiuser precoding. In: Wireless Communication Systems (ISWCS 2013), Proceedings of the Tenth International Symposium on (2013)

11. Lenstra, A.K., Lenstra, H.W., Lovász, L.: Factoring polynomials with rational coefficients. Mathematische Annalen 261(4), 515-534 (1982)

12. Luo, Y., Qiao, S.: A parallel LLL algorithm. In: Proceedings of The Fourth International C* Conference on Computer Science and Software Engineering, pp. 93-101 (2011)

13. Shabany, M., Youssef, A., Gulak, G.: High-throughput 0.13- $\mu \mathrm{m}$ CMOS lattice reduction core supporting $880 \mathrm{Mb} / \mathrm{s}$ detection. Very Large Scale Integration (VLSI) Systems, IEEE Transactions on 21(5) (2013)

14. Villard, G.: Parallel lattice basis reduction. In: Papers from the international symposium on Symbolic and algebraic computation, ISSAC '92. ACM, New York, NY, USA (1992)

15. Wetzel, S.: An efficient parallel block-reduction algorithm. In: Algorithmic Number Theory, pp. 323-337. Springer (1998)

16. Wu, D., Eilert, J., Liu, D.: A programmable lattice-reduction aided detector for MIMOOFDMA. In: Circuits and Systems for Communications, 2008. ICCSC 2008. 4th IEEE International Conference on, pp. 293-297 (2008)

17. Wubben, D., Seethaler, D., Jaldén, J., Matz, G.: Lattice reduction 28(3), 70-91 (2011) 